\title{
DRAFT: MATHEMATICAL AND NUMERICAL MODELLING OF WAVE IMPACT ON WAVE-ENERGY BUOYS
}

\author{
A. Kalogirou* \\ School of Mathematics \\ University of Leeds \\ LS2 9JT, Leeds, UK \\ Email: a.kalogirou@leeds.ac.uk
}

\author{
O. Bokhove \\ School of Mathematics \\ University of Leeds \\ LS2 9JT, Leeds, UK \\ Email: o.bokhove@leeds.ac.uk
}

\begin{abstract}
We report on the mathematical and numerical modelling of amplified rogue waves driving a wave-energy device in a contraction. This wave-energy device consists of a floating buoy attached to an AC-induction motor and constrained to move upward only in a contraction, for which we have realised a working scale-model. A coupled Hamiltonian system is derived for the dynamics of water waves and moving wave-energy buoys. This nonlinear model consists of the classical water wave equations for the free surface deviation and velocity potential, coupled to a set of equations describing the dynamics of a wave-energy buoy. As a stepping stone, the model is solved numerically for the case of linear shallow water waves causing the motion of a simple buoy structure with $V$-shaped cross-sections, using a variational (dis)continuous Galerkin finite elementmethod.
\end{abstract}

\section{INTRODUCTION}

The study of water waves has attracted scientists for decades, especially due to their effect on ships, offshore structures and oil exploitation. The interaction of waves can often lead to extreme wave phenomena, e.g. waves with irregular height. Waves with anomalously high amplitudes relative to the ambient waves are called rogue waves and can appear either at the coast or in the open ocean. Such waves can have significant impact on moving ships and wave-energy devices, and the main goal of our study is to investigate the effect of rogue waves on such floating

\footnotetext{
* Address all correspondence to this author.
}

bodies.

A water wave model is derived based on potential flow theory, a famous mathematical method in the modelling of water waves which describes the fluid velocity as the gradient of a scalar field: the velocity potential. Here we remain entirely within a variational framework $[1,2]$ in order to ensure zero numerical damping, important for wave propagation. The potential flow water wave model is coupled to a moving wave-energy buoy. This nonlinear model consists of the classical water wave equations for the free surface deviation and velocity potential, coupled to a set of equations describing the dynamics of the wave-energy buoy. The developed wave-buoy system is an essential step towards advancing in the modelling of wave impact on ships, as such an extension is straightforward. We explain this further in the discussion.

Similar fluid-structure interaction models using variational methods have been reported by other researchers $[3,4,5,6]$. In this paper, we also present numerical solutions based on a variational (dis)continuous Galerkin finite element method. The numerical implementation employs 2nd-order continuous Lagrange polynomial approximations in space and a 2nd-order discontinuous Störmer-Verlet symplectic scheme in time [7]. We obtain numerical results for the dynamics of the coupled system linearised around a rest state. In this case the problem becomes linear and we solve the system in the shallow water approximation, i.e. the dynamics are reduced to those on the free surface. Considering the shallow water limit is a stepping stone because the fundamentals of the wave-buoy coupling actually lie at the free surface 


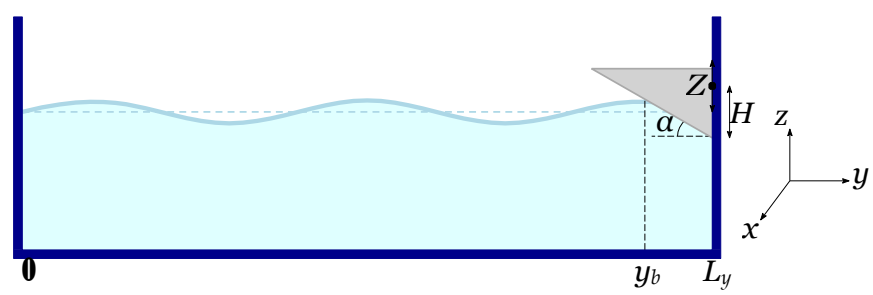

FIGURE 1: PROBLEM CONFIGURATION WITH A LINEAR BUOY AT THE RIGHT END OF THE WAVE TANK. THE POINT DENOTED BY $y_{b}$ IS THE FAR-LEFT POINT WHERE THE WATER MEETS THE BUOY.

and not the interior of the fluid, therefore the extension to potential flow then becomes straightforward. For simplicity, we also consider a simple buoy structure consisting of V-shaped crosssections; such a buoy structure is important because it can be easily extended to become the bow of a ship. Numerical results are first obtained for a problem where waves are generated by temporarily removing a sluice gate in a rectangular wave channel (see also [8] for a related problem concerning small-amplitude and long water waves). Finally, we present results for waves generated by a piston wavemaker in a wave channel that employs geometric wave amplification to create rogue-wave effects; such waves are in general nonlinear, but here we concentrate on linear wave impact on wave-energy buoys.

\section{MATHEMATICAL MODEL Model For Wave-Energy Buoy}

We consider three-dimensional water wave dynamics coupled to the dynamics of a wave-energy buoy of mass $M$. This buoy is restricted to move only in the vertical direction and its motion can be described by the position of its centre of mass $Z$ and the conjugate velocity $W$. We assume that the buoy has a simple linear hull structure, given by

$$
h_{b}(x, y ; t)=Z(t)-H-\tan \alpha\left(y-L_{y}\right) \text {, }
$$

as demonstrated in Fig. 1. Here, $\boldsymbol{\alpha}$ is the angle between the hull and the horizontal, $L_{y}$ is the length of the domain and $H>0$ is the vertical distance between the centre of mass $Z$ and the keel of the buoy (the keel of this V-shaped buoy resides at $y=L_{y}$ ). The point $y_{b}$ shown in the figure is defined as the far-left point where the water meets the buoy.

We should note that the buoy we considered initially was symmetrically extended to the right such that the point $Z$ was indeed the centre of mass of the triangular (or tetrahedral) body; we then decided to consider only "half" of the domain of interest because of the symmetry of the buoy, but kept the point $Z$ at the original position of the centre of mass.

\section{Variarional Principle For Shallow Water}

The classical potential flow model describes the evolution of the free surface $h(x, y, t)$ and the velocity potential $\varphi(x, y, z, t)$, where $x, y \in \Omega=\left[0, L_{x}\right] \times\left[0, L_{y}\right]$ are the horizontal spatial coordinates, $z \in[0, h(x, y, t)]$ is the vertical coordinate and $t \in[0, T]$ is time. Here we consider shallow water waves and this allows us to reduce the dynamics to the ones on the free surface, by seeking solutions for the free surface height $h(x, y, t)$ and the free surface velocity potential $\varphi_{s}(x, y, t)=\varphi(x, y, h(x, y, t), t)$. A variational principle for the dynamics of the wave-buoy system can be found by extending Luke' s variational principle [1] for shallow water waves and a buoy, and is given by

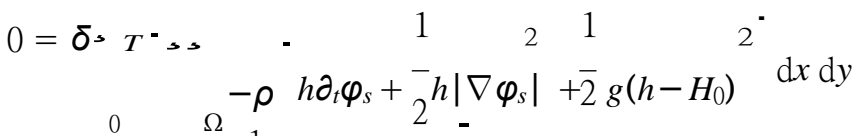

$$
\begin{aligned}
& +M W Z-\frac{1}{2} M W^{2}-M g Z \mathrm{~d} t \text {. }
\end{aligned}
$$

Here, $g$ is the gravitational force, $\rho$ is the density of water and $H_{0}$ is the flat water depth at rest. The gradient operator $\nabla=\left(\partial_{x}, \partial_{y}\right)^{T}$ is in the horizontal plane only.

\section{Linearisation}

The problem admits a steady state solution in the case when the water is flat and the buoy is floating at rest. The steady state solution is given by

$$
\begin{aligned}
& h=H_{0}, \quad \varphi=0, \quad h_{b}=H_{b}(y), \\
& Z=\bar{Z}, \quad W=0, \quad y_{b}=L_{b} .
\end{aligned}
$$

The waterline point $L_{b}$ has now a fixed position. The form of the hull of the buoy at the floating rest state is $H_{b}(y)=H_{b}(y ; \bar{Z})=$ $\bar{Z}-H-\tan \alpha\left(y-L_{y}\right)$, where $\bar{Z}=H_{0}+H->(2 M \tan \alpha) / \rho L_{x}$ is the rest position of the buoy' $s$ centre of mass. This is obtained by linearising the equations of motion (coming from (2)) and applying Archimedes' principle which indicates that the displaced volume of water should equal the mass of the buoy.

We linearise around the rest state (3) by adding small perturbations to the steady state solution, i.e.

$$
\begin{array}{lll}
h=H_{0}+\eta, & \varphi=\tilde{\varphi}, & h_{b}=H_{b}(y)+\eta_{b}, \\
Z=\bar{Z}+\tilde{Z}, & W=\tilde{W}, & y_{b}=L_{b}+\tilde{y}_{b} .
\end{array}
$$

A linear shallow water model can be then derived by considering only quadratic terms in the variational principle (in what follows, all tildes are dropped for simplicity and the subscript $s$ is omitted from the free surface potential). We first integrate the term $M W Z$ 
by parts and then change the sign of the quadratic variational principle, which is finally given by

$$
\begin{aligned}
& 0=\delta_{0} T_{\Omega_{w}} \leadsto{ }^{-} \rho \eta \partial_{t} \varphi+\frac{1}{2} \rho H_{0}|\nabla \varphi|^{2} \\
& +\rho g \cdot \frac{1}{-1} \eta^{2}-\eta_{R} \eta^{\cdot \cdot} \mathrm{d} x \mathrm{~d} y \\
& +{ }_{\Omega_{b}} \quad{ }^{-} \rho \eta_{b} \partial_{t} \varphi+\frac{1}{2} \rho H_{b}(y)|\nabla \varphi|^{2} \\
& +\rho g{\frac{1}{\eta^{2}}}_{\eta_{-}}^{2}-\eta_{R} \eta_{b}-\rho \lambda\left(\eta_{b}-Z\right) \stackrel{-}{\mathrm{d}} x \mathrm{~d} y \\
& +M Z W+\frac{1}{2} M W^{2} \mathrm{~d} t .
\end{aligned}
$$

Here we added a (gravitational) potential $\eta_{R}(y, t)$ to model a sluice gate release problem (more details in a later section). In the above variational principle, the spatial domain is split in two parts: in the water subdomain $\Omega_{w}, \eta$ is the usual surface deviation, while in the buoy subdomain $\Omega_{b}$, the water surface $\eta_{b}$ is constrained by the buoy. This condition is imposed by using a Lagrange multiplier $\lambda$, which enforces the constraint $\eta_{b}=Z$, essentially meaning that the water under the buoy takes exactly the shape of the wetted part of the buoy.

At the waterline point, we impose that the height of the fluid' $s$ free surface should be equal to the buoy' $s$ surface height, i.e. $h\left(x, y_{b}, t\right)=h_{b}\left(x, y_{b} ; t\right)$. In the linearised case, this becomes $\eta\left(x, L_{b}, t\right)=-\tan \alpha \tilde{y}_{b}+\tilde{Z}$. This condition hence provides an expression for the perturbation of the waterline point and is given by

$$
\tilde{y}_{b}=\frac{\tilde{Z}-\eta\left(x, L_{b}, t\right)}{\tan \alpha} .
$$

\section{Evolution Equations}

The final constrained system of evolution equations emerges from the variational principle (5) by applying the variations with respect the $\eta, \varphi, Z, W, \lambda$ and is given by

$$
\partial_{t} \varphi+g\left(\eta-\eta_{R}\right)-\lambda \Theta\left(y-L_{b}\right)=0, \text { in } \Omega,
$$

$\partial_{t} \eta+\nabla \cdot\left(\left(H_{0} \Theta\left(L_{b}-y\right)+H_{b} \Theta\left(y-L_{b}\right)\right) \nabla \varphi_{b}\right)=\chi$, in $\Omega_{b}$, ( ( ( $b$ b)

$$
\begin{array}{r}
M W+\rho \Omega_{\Omega_{b}}^{s} \lambda \Theta(y-L) \mathrm{d} x \mathrm{~d} y=0, \text { in } \Omega, \\
{ }^{b} \quad Z=W . \quad b
\end{array}
$$

In the above equations, $\Theta\left(y-L_{b}\right)$ is a heavyside step function such that $\Theta\left(y-L_{b}\right)=0$ if $y \leq L_{b}$ and $\Theta\left(y-L_{b}\right)=1$ if $y>L_{b}$. Equation (7c) is the constraint imposed in Eqn. (5) by use of the

\section{(DIS)CONTINUOUS GALERKIN DISCRETISATION Finite Element Discretisation In Space}

We expand the variables $\eta, \varphi$ and $\lambda$ in terms of continuous basis functions $\phi_{k}(x, y)$, such that

$$
\begin{gathered}
\eta \approx \eta_{h}=\eta_{k}(t) \phi_{k}(x, y), \quad \varphi \approx \varphi_{h}=\varphi_{k}(t) \phi_{k}(x, y), \\
\lambda \approx \lambda_{h}=\lambda_{k} \phi_{k}(x, y),
\end{gathered}
$$

where the Einstein summation convention is adopted. The total number of nodes will be denoted by $k, A$, the nodes at the free water surface by $\hat{k}, \hat{A}$ (including nodes at $y=L_{b}$ ) and the nodes at the buoy' s surface by $k, A$. Substituting these finite element expansions in Eqn. (5) and introducing the matrices

$$
\begin{aligned}
& M_{k A}={ }_{\Omega}^{s} \phi_{k} \phi_{A} \mathrm{~d} x \mathrm{~d} y, \quad \tilde{M}_{\tilde{k} \tilde{A}}={ }_{\Omega_{b}}^{s s} \phi_{k} \phi_{\tilde{A}} \mathrm{~d} x \mathrm{~d} y, \\
& Q_{A}={ }_{\Omega_{b}}^{\infty} \phi_{A} \mathrm{~d} x \mathrm{~d} y, \quad R_{k}(t)={ }_{\Omega}^{\infty} \eta_{R}(y, t) \phi_{k} \mathrm{~d} x \mathrm{~d} y, \\
& A_{k A}={ }_{\Omega_{w}} H_{0} \nabla \phi_{\hat{k}} \cdot \nabla \phi_{\hat{A}} \mathrm{~d} x \mathrm{~d} y+{ }_{\Omega_{b}} H_{b}(y) \nabla \phi_{\hat{k}} \cdot \nabla \phi_{\tilde{A}} \mathrm{~d} x \mathrm{~d} y,
\end{aligned}
$$

yields the time continuous but space discrete variational principle

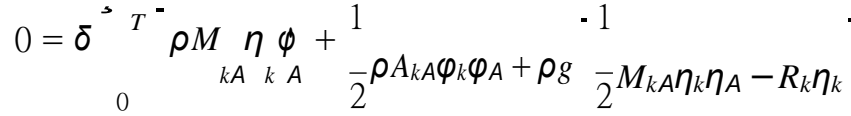

$$
\begin{aligned}
& -\rho\left(\tilde{M}_{k \tilde{A}} \eta_{\tilde{k}} \lambda_{\tilde{A}}-Q_{\tilde{A}} \lambda_{\tilde{A}} Z\right)+M Z \dot{W}+\frac{1}{2} M W^{2} \mathrm{~d} t . \quad(10)
\end{aligned}
$$

\section{Time Discretisation}

The variational principle in (10) is discretised in time using the RATTLE symplectic algorithm [9], which is an extension of the (discontinuous) symplectic Störmer-Verlet method for constraint Hamiltonian systems [10, 11, 12]. The final space-time discrete equations of motion are the following

$$
\begin{aligned}
& M_{k A} \varphi_{A}^{n+1 / 2}=M_{k A} \varphi_{A}^{n} \\
& -\frac{\Delta t^{*}}{2} g\left(M_{k A} \eta_{A}^{n}-R_{k}^{n+1 / 2}\right)-\widetilde{M}_{k \hat{A}} \lambda_{\tilde{A}}^{n+1 / 2^{-}}, \\
& M W^{n+1 / 2}=M W^{n} \quad \underline{\Delta t} \rho \underset{A}{Q} \lambda_{A^{-}}^{n+1 / 2} \text {, } \\
& M_{k A} \eta^{n+1} \quad{ }_{k A k}{ }^{-}{ }_{k A}{ }^{n+1 / 2} \\
& { }_{k}=M \eta^{n}+\Delta t A^{k A} \varphi_{k}, \\
& Z^{n+1}=Z^{n}+\Delta t W^{n+1 / 2} \text {, } \\
& 0=\tilde{M}_{\tilde{k} \tilde{A}} \eta_{\tilde{k}}^{n+1}-Q_{A} Z_{n+1}, \\
& M_{k A} \varphi_{A}^{n+1}=M_{k A} \varphi_{A}{ }_{n+1 / 2}^{\tilde{k}} \\
& \begin{aligned}
A & \left.-{ }^{g(M} \eta^{n+1}-R^{n+1 / 2}\right) \\
& k A A_{\Delta t}-{ }_{k \tilde{A} \tilde{A}} \lambda^{n+1 / 2^{-}} \\
M W^{n+1} & =M W^{n+1 / 2}-{ }^{k} \rho Q^{\sim}{ }_{n+1 / 2}
\end{aligned}
\end{aligned}
$$


Lagrange multiplier $\lambda$. 
We first solve for the Lagrange multiplier $\lambda_{A}^{n+1 / 2}$, i.e. we solve the constraint equation (11e). The Lagrange multiplier is determined by substituting the updates $\eta_{k}^{n+1}$ and $Z^{n+1}$ from (11c) and (11d), respectively, into the discrete constraint (11e), leading to a linear system for this Lagrange multiplier This is given below, where we have defined $\mu_{A^{n}}^{r+t z}=\frac{\Delta t}{2} \lambda^{n+1 / 2}$ for convenience,

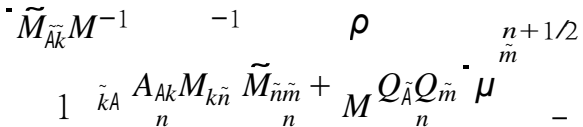

$$
\begin{aligned}
& ={ }_{\Delta t}\left(Q_{A} Z^{n}-\tilde{M}_{\tilde{k} \tilde{A}} \eta_{\tilde{k}}^{n}\right)+Q_{\tilde{A}}{ }^{n}+\tilde{M}_{\tilde{A} k} M_{\tilde{k} A}{ }^{1} A_{A k} \cdot{ }^{-} \frac{\Delta t}{2} g \eta_{k}-\varphi_{k}{ }^{n} .
\end{aligned}
$$

Notice that as the enforced constraint is relevant to the region under the buoy only, any sluice gate contribution cancels out in the above equation.

Having obtained the solution for $\mu_{A^{n}}^{n+1 / 2}=\frac{\Delta t}{2} \lambda_{A}^{n+1 / 2}$ by solving the constraint equation, the rest of the equations in the system (11) are then explicit and are solved in the following order

$$
\begin{aligned}
& M_{k A} \varphi^{n+1 / 2} \quad{ }_{\Delta}^{n} t^{-} \quad n \quad n+1 / 2 \\
& A=M_{k A} \varphi_{A+1 / 22} g\left(M_{k A} \eta_{A}-R_{k} \quad\right) \\
& +\tilde{M}_{k \tilde{A}} \mu_{\tilde{A}} \rho \\
& \begin{array}{l}
W^{n+1 / 2}=W^{n}-{ }_{n}{ }_{M}{ }_{A} \mu_{A^{\tau}}^{n+1 / 2}, \\
M_{k A} \eta^{n+1}
\end{array}
\end{aligned}
$$

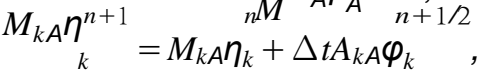

$$
\begin{aligned}
& Z^{n+1}=Z^{n}+\Delta t W^{n+1 / 2} \\
& M_{k A} \varphi^{n+1}=M_{k A} \varphi_{n+1 / 2}^{n+1 / 2}-{ }^{\Delta^{\prime} t} g\left(M_{k A} \eta_{A}^{n+1}-R_{k}^{n+1 / 2}\right) \\
& +\tilde{M}_{k \tilde{A}} \mu_{\tilde{A}} \\
& W^{n+1}=W^{n+1 / 2}-\frac{\rho}{M}{ }^{A}{ }_{A} \mu_{n+1 / 2} \text {. }
\end{aligned}
$$

\section{NUMERICAL SIMULATIONS \\ Waves Generated By Removing A Sluice Gate}

We consider a wave generated by removing a sluice gate after a finite time $T_{s}$ (see also [8] for a related water wave problem). We solve the linear shallow water equations coupled to a buoy, with initial condition $\varphi=0$ and $\eta=\eta_{R}$, where $\eta_{R}$ is defined by

$$
\begin{aligned}
& \sqcup h_{1}-h_{y} \frac{\left(T_{s}-t\right)}{T_{s}} \quad \text { if } y<y_{1} \\
& \begin{array}{cl}
\eta_{R}(y, t)={ }^{)}\left(h_{1}-h_{0}\right){\frac{\left(T_{s}-t\right)}{T_{s}}}^{-} 1-{\frac{y-y_{1}}{y_{2}-y_{1}}}^{-} & \text {if } y_{1} \leq y \leq y_{2} \\
\square_{0} & \text { if } y>y_{2},
\end{array}
\end{aligned}
$$

for $t<T_{s}$ and $\eta_{R}=0$ for $t \geq T_{s}$. Here, $0<y_{1}<y_{2}<L_{y}$, the
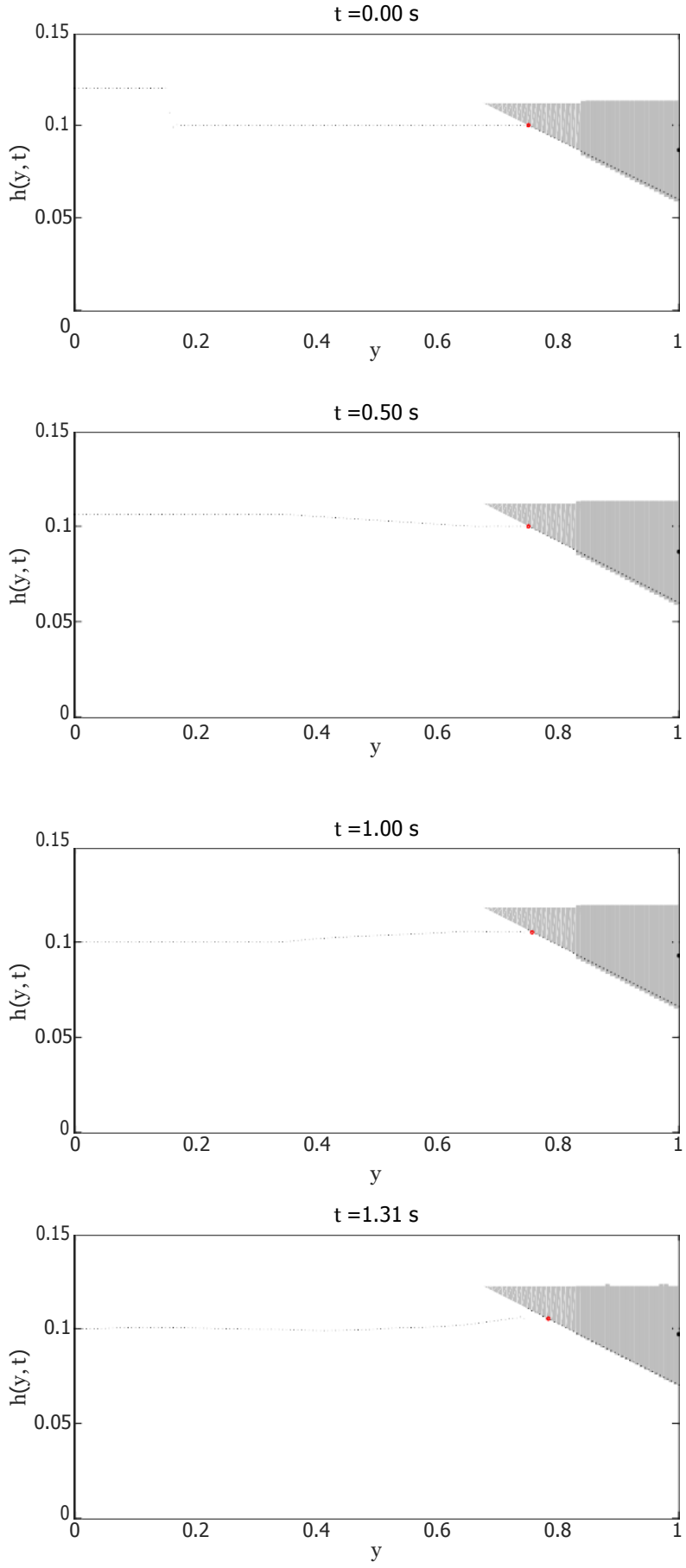

FIGURE 2: SNAPSHOTS OF THE NUMERICALLY COM-

PUTED WAVE HEIGHT $h(x, y, t)$ AT $x={ }_{2} L_{x}$ AND FOR $t=$ $0.0,0.5,1.0,1.31 \mathrm{~s}$. THE BLACK DOT AT $y=L_{y}$ SYMBOLinitial water level difference is $h_{1}-h_{0}>0$ at $t=0$ and $T_{s}>0$ is the sluice gate release time. In the numerical simulation, we take 
ISES THE POSITION OF $Z$ AND THE RED CIRCLE DENOTES THE WATERLINE POSITION. 

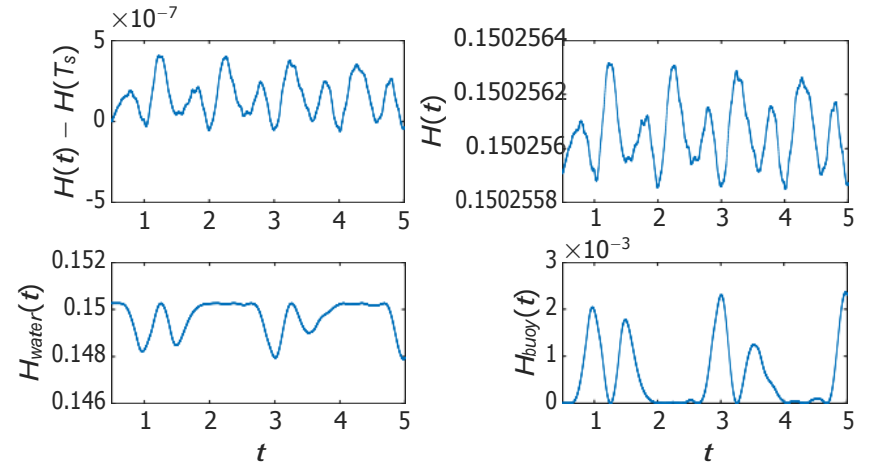

FIGURE 3: ENERGY OF THE PROBLEM WITH A REMOVABLE SLUICE GATE. THE TOTAL ENERGY $E(t)$ IS CONSERVED (TOP RIGHT PANEL) AND HAS SMALL VARIATIONS FROM THE "INITIAL" ENERGY $E\left(T_{s}\right)$, CALCULATED AT THE TIME WHEN THE SLUICE GATE IS COMPLETELY REMOVED (TOP LEFT PANEL). THE BOTTOM ROW ILLUSTRATES THE ANTICIPATED EXCHANGE OF ENERGY BETWEEN WATER (BOTTOM LEFT PANEL) AND BUOY (BOTTOM RIGHT PANEL).

$y_{1}=0.15 \mathrm{~m}, y_{2}=0.16 \mathrm{~m}$, lower and upper water levels $h_{0}=H_{0}$, $h_{1}=1.2 H_{0}$ and a sluice gate release time $T_{s}=0.5 \mathrm{~s}$.

The parameter values used in the numerical simulation are the following: the channel is rectangular, with width $L_{x}=1 \mathrm{~m}$, length $L_{y}=1 \mathrm{~m}$ and height $L_{z}=0.2 \mathrm{~m}$. Water fills half the height of the channel, i.e. the flat water depth is $H_{0}=0.1 \mathrm{~m}$. On the right end of the channel there a buoy of mass $M=5 \mathrm{~kg}$ and occupying about $1 / 4$ of the channel, i.e. the location of the waterline is at $L_{b}=0.75 \mathrm{~m}$ at the rest state. The angle $\alpha$ is computed via the relation $\alpha=\tan ^{-1}\left(2 M / \rho L_{x}\left(L_{y}-L_{b}\right)^{2}\right)$. The rest of the parameters are taken equal to their physical values, i.e. $g=9.81$ $\mathrm{m} / \mathrm{s}^{2}$ and $\rho=997 \mathrm{~kg} / \mathrm{m}^{3}$.

The numerical calculation is performed on a quadrilateral mesh with $N_{x}=10$ and $N_{y}=160$ elements in each horizontal dimension and linear polynomial approximation in each element,

i.e. a total of $N_{k}=N_{x} N_{y}=1600$ elements and $N_{n}=\left(N_{x}+1\right)\left(N_{y}+\right.$ 1) ${ }_{1}=1771$ modes. The time step was calculated sych that to satmerical simulations were first executed on MATLAB (based on our own stand-alone code) and then also using the automated system Firedrake [13], with equal results.

The outcome of the numerical simulation is demonstrated in Fig. 2, for a sluice gate and buoy uniform in $x$. The wave height at the middle of the cross-section $x=\frac{1}{2} L_{x}$ is shown at four different times $t=0.0,0.5,1.0,1.31 \mathrm{~s}$ (see also [14] for a video of the simulation). We can see that the removal of the sluice gate generates a single wave, which travels along the channel until it reaches the other end. It is then reflected on the right wall, si-

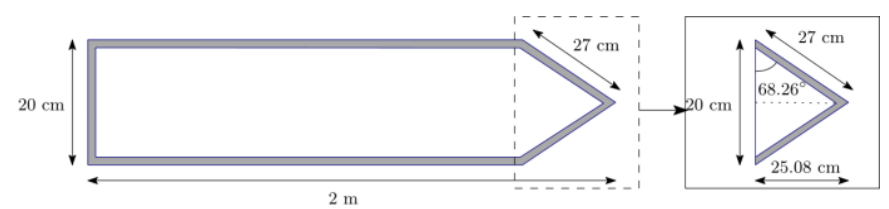

FIGURE 4: SKETCH OF THE TOP-VIEW OF A WAVE TANK WITH A CONTRACTION AT THE RIGHT END.

multaneously causing the buoy to shift upwards. The waterline point is plotted according to relation (6) and follows an anticipated motion, i.e. it moves higher when the wave hits the buoy and then moves to a lower position to the right when the buoy is shifted upwards.

Figure 3 illustrates the behaviour of the energy of the system. It is clear that the total energy $E(t)$ is conserved, in the sense that it is bounded and shows no drift - it is not increasing or decreasing in time - and has small variations from the "initial" energy $E\left(T_{s}\right)$ (found as soon as the sluice gate is completely removed). The deviation $E(t)-E\left(T_{s}\right)$ is of the order of $10^{-7}$ for the simulation presented here, and we have confirmed that this error is reduced by $(\Delta t)^{2}$ since we employ a second-order numerical method in time. Moreover, at the beginning of the simulation the buoy is motionless and has zero energy. When the water wave reaches the right end area of the wave tank, it causes the vertical motion of the buoy and thus it loses energy which converts to kinetic energy of the buoy. This exchange of energy is such that the total energy is conserved, as expected.

\section{Waves Generated By A Piston Wavemaker}

We now consider that a wave is generated by a (linearised) wavemaker located at the left side of the channel, i.e. at $y=$ $R(t) \approx 0$, with $R(t)=\frac{A}{\omega}(1-\cos (\omega t))$. The channel has a Vshaped contraction at the other end (Fig. 4) and in the corner there is a wave-energy buoy limited to move only in the vertical. We solve a set of modified linear shallow water equations coupled with the buoy, resulting from a variation ${ }_{\text {(5) }}$ but winciple similar to $\eta_{R}=0$ and the term $\rho H_{0}$ added on the left boundary. The corresponding term in the space-time discrete evolution equations (11) is

$$
\left.\Delta t H_{0} \frac{\mathrm{d} R}{\mathrm{~d} t}\right|_{t_{n}+1 / 2} W_{k} \text {, with } W_{k}=\left.{ }_{0}^{s L_{x}} \phi_{k}\right|_{y=0} \mathrm{~d} x,
$$

which appears on the right hand side of Eqn. (11c). Moreover, note that in this case the rest position of the buoy' s centre of mass is

$$
\bar{Z}=H_{0}+H-3 \frac{-3 M \tan \theta_{c}(\tan \alpha)^{2}}{\rho},
$$




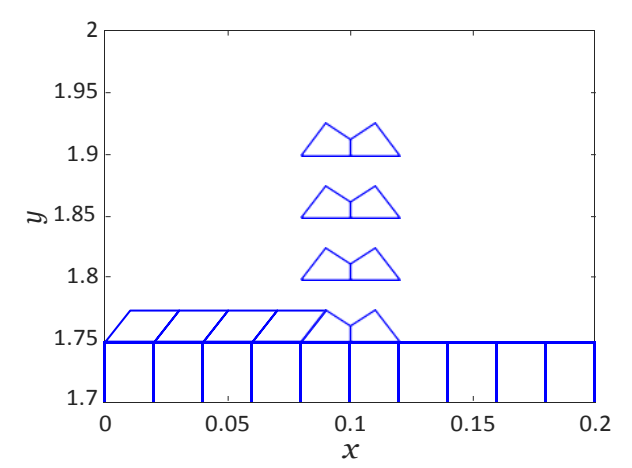

FIGURE 5: SAMPLE MESH STRUCTURE IN THE CONTRACTION FOR $N_{x}=10$ AND $N_{y}=30$. THE ADDITIONAL NODES IN THE CONTRACTION ARE DENOTED WITH A RED $\times$.

and $\alpha=\tan ^{-1}\left(3 M \tan \theta_{c} / \rho\left(L_{y}-L_{b}\right)^{3}\right)$. The angle $\theta_{c}=68.26^{\circ}$ is the angle between the opening of the contraction and the vertical (see also the magnified right hand side panel in Fig. 4).

The system is solved with initial conditions $\varphi=0$ and $\eta=0$. The wavemaker starts from rest and moves only in the horizontal with an amplitude of $A=0.0284 \mathrm{~m}$ and frequency $\omega=\frac{8 \pi}{L y} \overline{g H_{0}}$ (this corresponds to a physical frequency of approximately $2 \mathrm{~Hz}$ ). The rest of the parameter values used in this numerical simulation are the following: the channel has dimensions $L_{x}=0.2 \mathrm{~m}$, $L_{y}=2 \mathrm{~m}$ and $L_{z}=0.2 \mathrm{~m}$. The initial constant water depth is $H_{0}=0.1 \mathrm{~m}$. In the contraction of the channel there a buoy of mass $M=0.05 \mathrm{~kg}$, with $L_{b}=1.9 \mathrm{~m}$ at the rest state. The mesh is assembled by uniform quadrilateral elements up to the entrance of the contraction, with $N_{x}=20$ and $N_{y}=175$. In the contraction, a quadrilateral mesh is still formed but the nodes are only aligned in every other line (see Fig. 5). In total, $N_{k}=3710$ elements are used, with $N_{n}=3916$ nodes. The time step used is $\Delta t=0.0011$.

The numerically computed wave height and the motion of the buoy in the contraction can be seen in Fig. 6 at $t=$ 1.0, 2.0, 2.2, $2.36 \mathrm{~s}$ (a video of the simulation can be found in [14]). The waterline is again marked with red circles. The motion of the buoy in this example is more complicated and this is because several waves are generated by the wavemaker, which not only interact with each other after reflection at the end of the channel, but are also amplified after entering the contraction. Furthermore, the velocity potential at $t=2.5 \mathrm{~s}$ is portrayed in Fig. 7. We note that the distinctive structure of the potential at the right end in the figure is due to the presence of the buoy.

In this numerical simulation, the total energy $E(t)$ increases with time due to the motion of the wavemaker (Fig. 8), which essentially introduces extra kinetic energy into the system. The energy is expected to be conserved if the wavemaker is switched
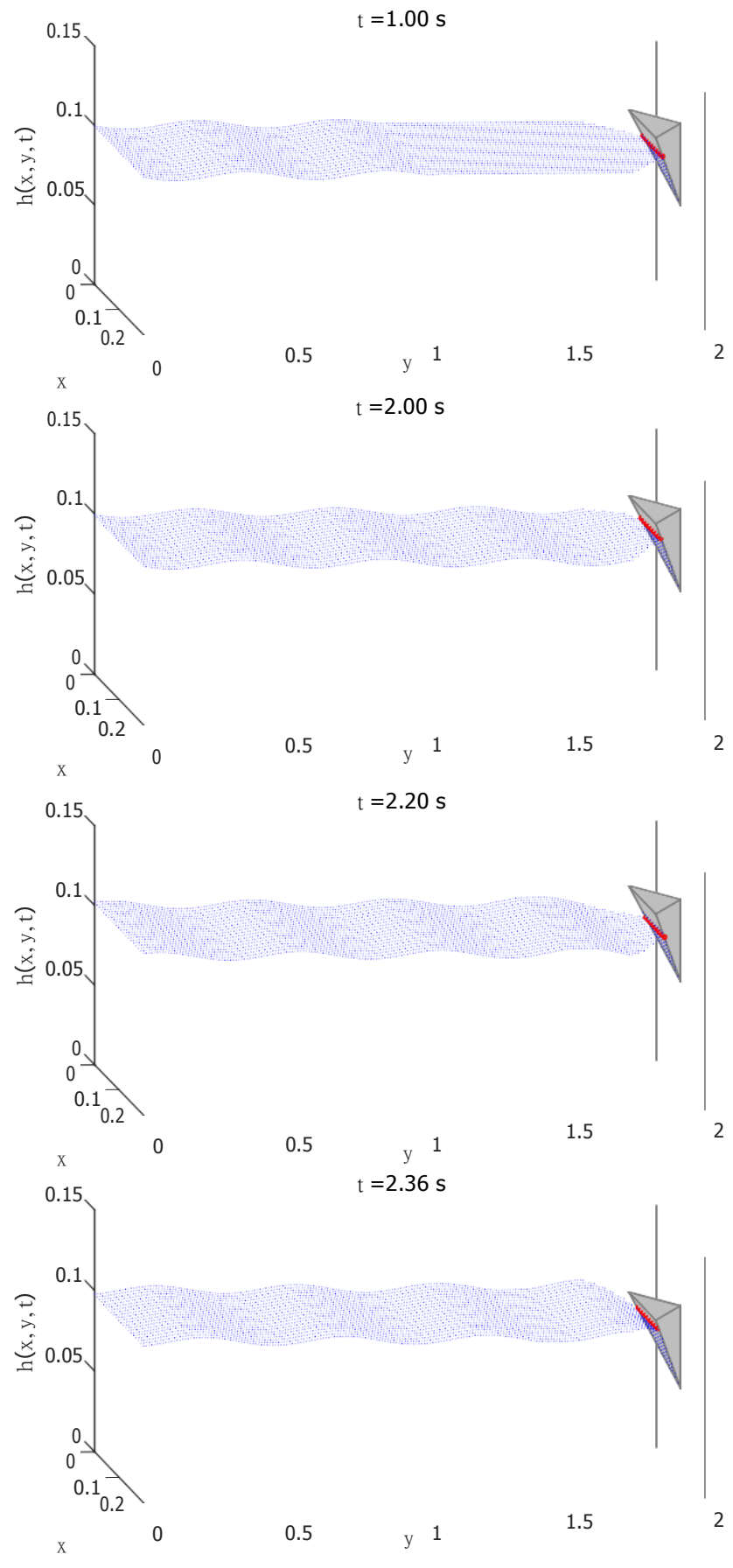

FIGURE 6: SNAPSHOTS OF THE NUMERICALLY COMPUTED WAVE HEIGHT $h(x, y, t)$ FOR $t=1.0,2.0,2.2,2.36 \mathrm{~s}$, IN A WAVE CHANNEL WITH A V-SHAPED CONTRACTION AT ONE END. THE RED CIRCLES DENOTE THE WATERLINE. 


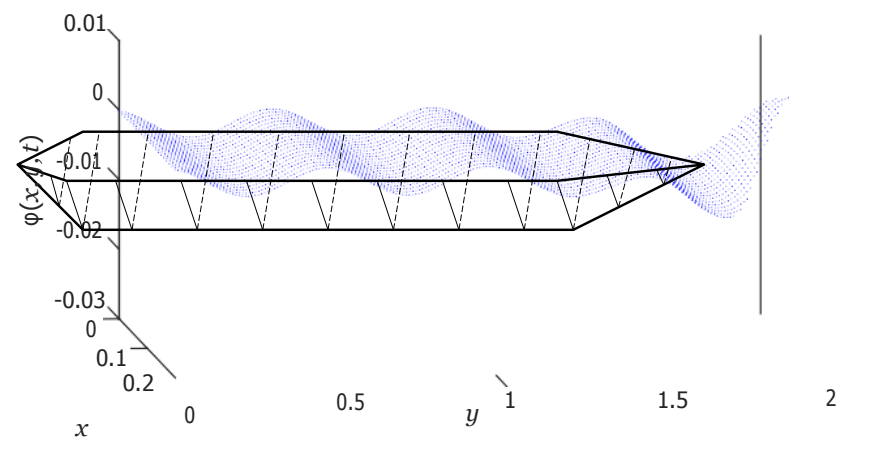

FIGURE 7: VELOCITY POTENTIAL AT $t=2.5 \mathrm{~s}$. THE POTENTIAL HAS A DIFFERENT STRUCTURE IN THE REGION UNDER THE BUOY.
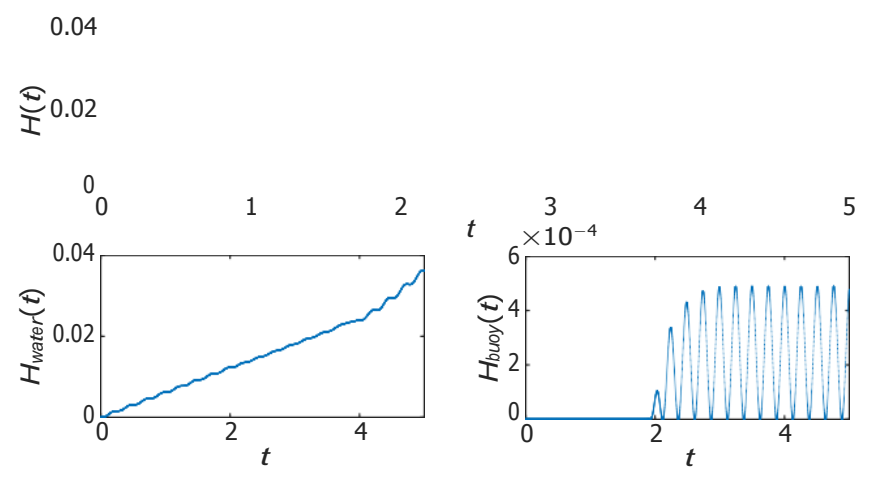

FIGURE 8: ENERGY OF THE PROBLEM WITH A PISTON WAVEMAKER. THE TOTAL ENERGY $E(t)$ IS NOT CONSERVED IN THIS CASE (TOP PANEL) BECAUSE ENERGY IS INJECTED IN THE SYSTEM DUE TO THE MOTION OF THE WAVEMAKER.

off after a few oscillations, but this was not verified in this study (cf. [8] for an alternative technique to prove "energy" conservation for non-autonomous systems).

\section{DISCUSSION}

The motion of a wave-energy buoy in a long wave channel with a contraction is studied by variational modelling and numerical simulations. The wave-energy buoy is constrained in the contraction of the channel and is allowed to move only in the vertical. We present a mathematical model based on potential flow theory, which describes the dynamics of shallow water waves and couples them to the dynamics of the buoy. This model is Hamiltonian and arises through an extension of Luke' s variational principle [1] to include the buoy. The final equations of

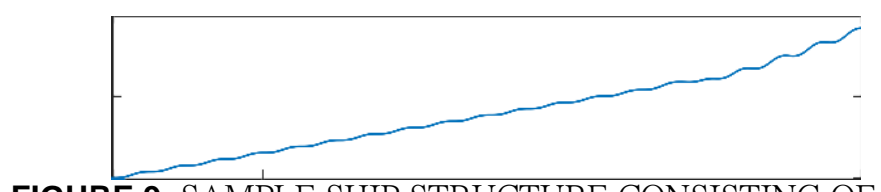

FIGURE 9: SAMPLE SHIP STRUCTURE CONSISTING OF V-SHAPED CROSS-SECTIONS.

motion are linearised around a steady state and consist of the classical shallow water wave equations for the free surface deviation and velocity potential, coupled to appropriate equations for the dynamics of the wave-energy buoy. The derived system of evolution equations includes a Lagrange multiplier $\lambda$, imposing the constraint that the fluid surface under the buoy takes its exact shape. Our shallow water theory can be easily extended to the full potential flow, i.e. for the dynamics of the whole fluid and not only its free surface. Such an extension to include the interior dynamics is straightforward, since the essentials of the coupling lie at the free surface; this is currently work in progress and will be used in comparisons of long-wave solutions with the present shallow water results.

The model is discretised using a continuous Galerkin finite element method for the spatial discretisation, and for the time discretisation a (dis)continuous symplectic scheme for constraint systems is applied. Numerical results are demonstrated for two cases: (i) waves generated by removing a sluice gate in a rectangular channel with a buoy uniform in $x$, and (ii) waves generated by the sinusoidal motion of a (linearised) wavemaker in a channel with a contraction and a tetrahedral buoy. The results confirm conservation of energy of the coupled system in case (i), after the sluice gate is completely removed. Moreover, even though the geometry of the channel used in case (ii) allows the generation of nonlinear rogue waves, one needs to follow the dynamics in the nonlinear regime in order to study rogue-wave impact on wave-energy buoys.

In addition, the mathematical model presented here can be extended in a straightforward manner for the case of a ship. The similarity of the buoy' s shape to the bow of a ship, allows us to consider a simple ship structure consisting of V-shaped cross sections, similar to the one portrayed in Fig. 9. Such a ship is allowed to move in the three-dimensional space and has 6 degrees of freedom: the three translational motions (heave, sway, surge) and the three rotational motions (pitch, roll, yaw). Hence the dynamics of the ship are described by the position of centre of mass $X=(X, Y, Z)$ and the three rotational angles $\theta=(\theta, \phi, \psi)[15]$. In addition, the conjugate variables are the velocity of the centre of mass $U=(U, V, W)$ and the angular momenta $p=\left(p_{\theta}, p_{\phi}, p_{\psi}\right)$. The ship has mass $M$ and moments of inertia $I=\left(I_{1}, I_{2}, I_{3}\right)$ (and angular velocities $\Omega=\left(\Omega_{1}, \Omega_{2}, \Omega_{3}\right)$ ), which are known. An extension of the variational principle (2) 


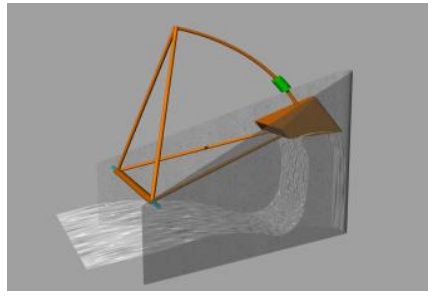

(a)

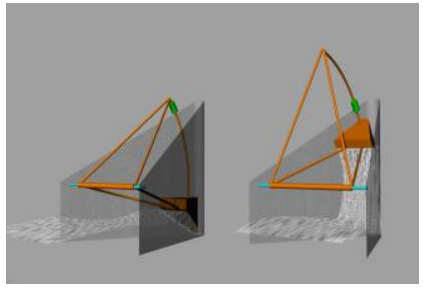

(b)
FIGURE 10: THREE-DIMENSIONAL BUOY IN A WAVE TANK CONTRACTION, ATTACHED TO AN ACINDUCTION MOTOR [16].

to include the dynamics of this ship is

$$
\begin{aligned}
0=\delta & T^{T_{s}}-\rho-\rho_{h} \varphi_{s}+\frac{1}{2} h\left|\nabla \varphi_{s}\right|^{2}+\frac{1}{2} g\left(h-H_{0}\right)^{2} \mathrm{~d} x \mathrm{~d} y \\
& 0 \quad \Omega \\
& +M U \cdot X+p \cdot \theta-\frac{1}{2} M|U|^{2}-\frac{1}{2}(I \otimes \Omega) \cdot \Omega-M g Z \quad \mathrm{~d} t .
\end{aligned}
$$

Here, symbol $\otimes$ is the Kronecker product defined by $I \otimes \Omega=$ $\left(I_{1} \Omega_{1}, I_{2} \Omega_{2}, I_{3} \Omega_{3}\right)^{T}$. The above wave-ship model is the subject of ongoing work and numerical computations are currently in progress; the results will be reported elsewhere.

Finally, we intend to perform experiments in a wave tank that employs geometric wave amplification through a linear Vshaped contraction and includes a wave-energy buoy in the corner of this contraction [16]. The buoy is attached to an ACinduction motor (as shown in Fig. 10) and is restricted to move only in the vertical. A study aimed at comparisons between our numerical results and the experiment is left as future work.

\section{ACKNOWLEDGMENT}

We acknowledge funding from Engineering and Physical Sciences Research Council (EPSRC) grant no. EP/L025388/1, with a link to the Dutch Technology Foundation STW for the project "FastFEM: behaviour of fast ships in waves" . Special regards to the Firedrake development team, in particular Lawrence Mitchell, David Ham and Colin Cotter, for assistance in using Firedrake.

\section{REFERENCES}

[1]Luke, J. C., 1967. "A variational principle for a fluid with a free surface” . J. Fluid Mech., 27, pp. 395 - 397.

[2]Miles, J. W., 1977. “On Hamilton' s principle for surface waves” . J. Fluid Mech., 83, pp. $153-158$. [3]van Daalen, E. F. G., van Groesen, E., and Zandbergen, P. J., 1993. "A Hamiltonian formulation for nonlinear wavebody interactions" . In Eighth International Workshop on Water Waves and Floating Bodies, IWWWFB, pp. 159 163.

[4]Kock, E., and Olson, L., 1991. "Fluid-structure interaction analysis by the finite element method-a variational approach” . Int. J. Numer. Meth. Eng., 31, pp. 463 - 491.

[5]Miloh, T., 1984. "Hamilton's principle, Lagrange' s method, and ship motion theory" . J. Ship Res., 28, pp. 229 237.

[6]Xing, J. T., and Price, W. G., 1997. "Variational principles of nonlinear dynamical fluid-solid interaction systems" . Phil. Trans. R. Soc. Lond. A, 355, pp. 1063 - 1095.

[7]Hairer, E., Lubich, C., and Wanner, G., 2006. Geometric numerical integration. Springer.

[8]Bokhove, O., and Kalogirou, A., 2016. "Variational water wave modelling: from continuum to experiment" . In Lectures on the Theory of Water Waves, T. Bridges, M. Groves, and D. Nicholls, eds., LMS Lecture Note Series. Cambridge University Press, United Kingdom.

[9]Andersen, H., 1983. "Rattle: A 'velocity' version of the shake algorithm for molecular dynamics calculations" . $J$. Comput. Phys., 52, pp. $24-34$.

[10]Cotter, C. J., Frank, J., and Reich, S., 2004. "Hamiltonian particle-mesh method for two-layer shallow-water equations subject to the rigid-lid approximation” . SIAM J.Appl. Dyn. Syst., 3, pp. $69-83$.

[11]Cotter, C. J., and Reich, S., 2004. Time stepping algorithms for classical mechanics. Tech. rep., Imperial College London. Available from: http: //wwwf.imperial.ac . uk/ sreich/03 6.pdf.

[12]Leimkūhler, B., and Skeel, R., 1994. " "Symplectic numerical integrators in constrained Hamiltonian systems".$J$. Comput. Phys., 112, pp. $117-125$.

[13]Markall, G. R., Rathgeber, F., Mitchell, L., Loriant, N., Bertolli, C., Ham, D. A., and Kelly, P. H. J., 2013. "Performance - portable finite element assembly using PyOP2 and FEniCS" . In Proceedings of the 28th Int. Supercomputing Conf., ISC, Kunkel, J. Martin, T. Ludwig, Meuer, and H. Werner, eds., Vol. 7905 of Lecture Notes in Computer Science. Springer, pp. $279-289$.

[14]Kalogirou, A., 2016. Water waves simulations and experiments. Available from: https://www. youtube. com/channel/UCUZAYHtVoiMqepeflisp $66 \mathrm{~g}$.

[15]Marsden, J. E., and Ratiu, T. S., 1994. Introduction to mechanics and symmetry, second ed. Springer-Verlag.

[16]Bokhove, O., and Zweers, W., 2013. RogueWavEnergy. Available from: http: / / www1. maths. leeds.ac . uk/ obokhove/WaveEnergy.m4v. 